\title{
URBAN-RURAL DIFFERENCES IN THE PREVALENCE OF MUSCLE WEAKNESS AND SLOW GAIT SPEED: A CROSS-SECTIONAL ANALYSIS FROM THE NHANES (2001-2002 AND 2011-2014)
}

\author{
J.J. Aziz', K.F. Reid', J.A. Batsis ${ }^{2}$, R.A. Fielding ${ }^{1}$
}

\begin{abstract}
Background: Older adults living in rural areas suffer from health inequities compared to their urban counterparts. These include comorbidity burden, poor diet, and physical inactivity, which are also risk factors for sarcopenia, for which muscle weakness and slow gait speed are domains. To date, no study has examined urban-rural differences in the prevalence of muscle weakness and slow gait speed in older adults living in the United States. Objective: To compare the prevalence of grip strength weakness and slow gait speed between urban and rural older adults living in the United States. Design: A cross-sectional, secondary data analysis of two cohorts from the National Health and Nutrition Examination Survey (NHANES), using gait speed or grip strength data, and urban-rural residency, dietary, examination, questionnaire and demographic data. Participants: 2,923 adults ( $\geq 60$ yrs.). Measures: Grip weakness was defined as either, an absolute grip strength of $<35 \mathrm{~kg}$. and $<20 \mathrm{~kg}$. or grip strength divided by body mass index (GripBMI) of $<1.05$ and $<0.79$ for men and women, respectively. Slow gait speed was defined as a usual gait speed of $\leq 0.8 \mathrm{~m} / \mathrm{s}$. Results: The prevalence of GripBMI weakness was significantly higher in urban compared to rural participants $(27.4 \%$ vs. $19.2 \%$; $\mathrm{p}=0.001)$, whereas their absolute grip strength was lower $(31.75( \pm 0.45)$ vs. 33.73( \pm 0.48$)$ ). No urban-rural differences in gait speed were observed. Conclusions: Older adults residing in urban regions of the United States were weaker compared to their rural counterparts. This report is the first to describe urban-rural differences in handgrip strength and slow gait speed in older adults living in the United States.
\end{abstract}

Key words: Sarcopenia, frailty, older adults, grip strength, gait speed.

\section{Introduction}

Sarcopenia, defined as the age-related loss of skeletal muscle mass and function, is associated with adverse health outcomes such as mobility disability, increased falls risk, frailty and death (1). Reports on the overall prevalence of sarcopenia vary widely (2), due largely to inconsistent definitions of sarcopenia (3). Several international working groups have attempted to better define sarcopenia. Recently, the Sarcopenia Definition and Outcomes Consortium (SDOC), found that measures of handgrip strength, independently, better discriminated mobility disability (slow gait speed) and distal health outcomes compared to measures of muscle mass (4).

Approximately $20 \%$ of older adults in the United States reside in rural areas (5). Individuals living in these areas have been reported to experience health inequities

1. Nutrition, Exercise Physiology and Sarcopenia Laboratory, Jean Mayer USDA Human Nutrition Research Center on Aging at Tufts University, Boston, MA, USA; 2. Associate Professor, Division of Geriatric Medicine and Gillings School of Global Public Health

Corresponding Author: Jason Aziz, Nutrition, Exercise Physiology and Sarcopenia Laboratory, Jean Mayer USDA Human Nutrition Research Center on Aging at Tufts University, 711 Washington St. Boston, MA 02111, USA e-mail: azizj@merrimack.edu

Received February 25, 2021

Accepted for publication March 19, 2021 compared to their urban counterparts (6), and are also at risk for higher healthcare costs. Indeed, rural adults in the United States rank poorly in 21 of 23 population health indicators, behaviors and risk factors (7), many of which are modifiable. Some of these health inequities, such as physical inactivity and poor nutrition status are associated with sarcopenia, thus it is plausible that the prevalence of sarcopenia may be higher in rural, compared to urban settings, however, no studies examining these differences exist. There is public health importance in identifying regional differences in the prevalence of sarcopenia and mobility limitations, so that precise, targeted interventions to prevent this condition may be developed. Therefore, the purpose of this study was to compare urban-rural differences in the prevalence of grip strength defined sarcopenia and slow gait speed in a population representative sample of communitydwelling older adults living in the United States, using the National Health and Nutrition Examination Survey (NHANES). We hypothesized that, because of health inequities between urban and rural older adults, the prevalence of sarcopenia and slow gait speed would be higher in rural, compared to urban populations. As a secondary aim, we evaluated associations between rural residency status and factors reported to be associated 
with indicators of sarcopenia.

\section{Methods}

We performed a cross-sectional, secondary data analysis, using the NHANES. This survey uses a complex multi-stage probability sampling design to derive a population-representative sample of non-institutionalized civilian citizens living in the United States. The NHANES collected gait speed data in 2001-2002 and handgrip strength test in 2011-2012 and 2013-2014. These data, along with urban-rural classification, examination, dietary, and questionnaire data were used in these analyses.

\section{Participants}

Because the NHANES did not collect gait speed and grip strength data in the same survey cycles, we created two separate analytic samples including of older adult men and women with non-missing urban-rural and nonmissing gait speed data (2001-2002; age range: 60-85 yrs.; $\mathrm{n}=1,350$; Gait Speed Cohort) or non-missing grip strength and anthropometric data (combined; 2011-2014; age range: $60-80$ yrs.; $n=1,573$; Grip Strength Cohort). This study was approved by Tufts University Social Behavior Educational Research (SBER) Institutional Review Board.

\section{Outcome measures}

Despite the fact that the SDOC recommends that slow gait speed and grip weakness are used to classify sarcopenia, the NHANES did not measure both parameters concomitantly, thus, we assessed each parameter in separate cohorts. Slow gait speed was defined as a usual gait speed of $\leq 0.8 \mathrm{~m} / \mathrm{s}$ (8). Grip weakness was defined as an absolute grip strength of $<35.5 \mathrm{~kg}$. and $<20 \mathrm{~kg}$. or grip strength divided by body mass index of $<1.05$ and $<0.79$ (GripBMI), in men and women, respectively (9).

\section{Urban-rural classification}

We used a binary urban-rural indicator variable to classify rural residency status. Rural residency classification was defined by the US Census Bureau which defines Urban Areas (UAs) as regions with 50,000 people or more and Urban Clusters (UCs) as regions with 2,500-50,000 people. Regions defined as 'rural' encompass all participants, housing and territory not included in a UA or UC (10).

\section{Examination data}

A 20' (6.1 meters) walk test was performed in the Mobile Examination Center (MEC). The participant was asked to walk at their usual pace. Start and stop times were defined as when the participant's first foot crossed the start line and when the participant's first foot crossed the 20' line. We report usual gait speed in meters per second. The handgrip strength test was performed in the standing position. Maximum handgrip strength was defined as the highest value among six possible bilateral attempts reported to the nearest kilogram. Body mass was measured on digital scale and reported in kilograms. Standing height was recorded using a stadiometer and reported in centimeters. More detailed descriptions of the measured walk and grip strength protocols in the NHANES has been published (11).

\section{Dietary Measures}

Participants completed an interviewer-assisted 24-hour diet recall in the MEC, using the automated multiple pass method (12). In NHANES 2011-2014, a second 24-hour recall was performed by telephone 3-10 days later. A detailed description of the NHANES dietary interview method has been described elsewhere (13). Total energy $(\mathrm{kcals} / \mathrm{d})$ and protein $(\mathrm{g} / \mathrm{d})$ intakes were primary nutrients of interest, as they have been associated with sarcopenia $(14,15)$, and also have been reported to vary between urban and rural populations (16). For the 20012002 dietary data, total energy and total protein were used, and for the 2011-2014 survey cycles, the withinperson means of day 1 and day 2 recalls for total energy and total protein were used. Total protein was adjusted for energy intake using the residuals method (17).

\section{Questionnaire data}

Age, sex, race/Hispanic origin and poverty to income ratio were collected from the demographic data file. To derive a measure of poverty status, we used the poverty to income ratio. In the NHANES, a family's income was divided by the current poverty guideline for that year. A value of $<1.0$ was defined as 'poor'. We identified the following medical conditions, as comorbidities reported to be associated with sarcopenia: heart disease (18), diabetes (19), chronic obstructive pulmonary diseases (20), arthritis (21) and obesity (22). In the Gait Speed cohort, participants were asked about muscle strengthening activities over the past 30 days. We considered those performing eligible activities two or more days per week on average, as 'strength trained'. In the Grip Strength cohort, we used minutes of sedentary activity as an inverse proxy measure for physical activity. Smoking status was classified as having never smoked, current smoker or former smoker. Health insurance status was self-reported coverage by any health insurance plan. 
Table 1

Participant baseline characteristics by urbanization status for each cohort: NHANES 2001-2002 and NHANES 2011-2014

\begin{tabular}{|c|c|c|c|c|c|c|c|c|}
\hline & \multicolumn{4}{|c|}{ NHANES 2001-2002 (Gait speed cohort) } & \multicolumn{4}{|c|}{ NHANES 2011-2014 (Grip strength cohort) } \\
\hline & \multirow{2}{*}{$\begin{array}{l}\text { Overall } \\
n=1,350\end{array}$} & \multirow{2}{*}{$\begin{array}{c}\text { Urban } \\
\mathrm{n}=\mathbf{1 1 7 6}(83.3 \%)\end{array}$} & \multirow{2}{*}{$\begin{array}{c}\text { Rural } \\
\mathrm{n}=174(16.7 \%)\end{array}$} & \multirow[t]{2}{*}{ p-value } & \multirow{2}{*}{$\begin{array}{l}\text { Overall } \\
n=1,573\end{array}$} & \multirow{2}{*}{$\begin{array}{c}\text { Urban } \\
\mathrm{n}=1,362(80.7 \%)\end{array}$} & Rural & \multirow[t]{2}{*}{ p-value } \\
\hline & & & & & & & $\mathrm{n}=211 \quad(19.4 \%)$ & \\
\hline Age & $70.69(0.29)$ & $70.84(0.28)$ & $69.94(1.32)$ & 0.522 & $69.31(0.29)$ & $69.29(0.30)$ & $69.40(0.69)$ & 0.876 \\
\hline Female & $688(57.4 \%)$ & $599(57.3 \%)$ & $(89) 57.9 \%$ & 0.893 & $809(54.1 \%)$ & $703(55.1 \%)$ & $106(49.9 \%)$ & 0.101 \\
\hline Race/Hispanic origin & & & & 0.006 & & & & $<0.001$ \\
\hline Mexican/Other Hispanic & $(235) 5.4 \%$ & $(226) 6.3 \%$ & (9)1.2\% & & $304(7.5 \%)$ & $292(8.9 \%)$ & $12(1.7 \%)$ & \\
\hline Non-Hispanic White & $(858) 83.5 \%$ & $(719) 81.7 \%$ & $(139) 92.8 \%$ & & $781(78.3 \%)$ & $608(74.6 \%)$ & $173(94 \%)$ & \\
\hline Non-Hispanic Black & $(227) 8.1 \%$ & $(202) 8.6 \%$ & $(25) 5.6 \%$ & & $342(9.1 \%) \%$ & $320(10.5 \%)$ & $22(3.6 \%)$ & \\
\hline Other & (30)2.9\% & (29)3.4\% & (1)0.5\% & & $146(5.1 \%)$ & $142(6.1 \%)$ & $4(0.7 \%)$ & \\
\hline BMI (kg./ m²) & $28.27(0.17)$ & $28.14(0.16)$ & $28.93(0.62)$ & 0.200 & $29.17(0.24)$ & $29.15(0.20)$ & $29.27(0.71)$ & 0.859 \\
\hline $\begin{array}{l}\text { Participates in strength } \\
\text { trainingt }\end{array}$ & $(215) 16 \%$ & (198)17.3\% & (17)9.5\% & 0.001 & -- & -- & -- & -- \\
\hline Sedentary time (min./day) & - & -- & - & -- & $430.17(6.42)$ & $438.34(8.45)$ & 396.01(18.35) & 0.070 \\
\hline$\#$ of co-morbidities $\ddagger$ & & & & 0.379 & & & & 0.615 \\
\hline 0 & (353) $27.2 \%$ & (314) $28.1 \%$ & (39)22.5\% & & $379(22.1 \%)$ & $339(22.3 \%)$ & $40(21.4 \%)$ & \\
\hline 1 & $(484) 37.6 \%$ & (423)36.9\% & $(61) 41.5 \%$ & & $527(34.9 \%)$ & $462(35.7 \%)$ & $65(32 \%)$ & \\
\hline 2 & (290)23.3\% & (253) $23.4 \%$ & $(37) 22.6 \%$ & & $435(28.6 \%)$ & $366(27.6 \%)$ & $69(32.7 \%)$ & \\
\hline$\geq 3$ & (133)12\% & (111)11.7\% & (22)13.5\% & & $232(14.4 \%)$ & $195(14.5 \%)$ & $37(13.9 \%)$ & \\
\hline Smoking status & & & & 0.174 & & & & 0.892 \\
\hline Never & $(649) 47.9 \%$ & $(557) 46.2 \%$ & (92)56.4\% & & $778(51.1 \%)$ & $673(50.9 \%)$ & $105(51.8 \%)$ & \\
\hline Current & $(160) 12 \%$ & (139)12.2\% & (21)11.2\% & & $195(10.4 \%)$ & $170(10.3 \%)$ & $25(10.9 \%)$ & \\
\hline Former & $(535) 40.1 \%$ & $(474) 41.6 \%$ & $(61) 32.4 \%$ & & $556(38.5 \%)$ & $485(38.7 \%)$ & $71(37.3 \%)$ & \\
\hline Covered by health insurance & $1299(96.3 \%)$ & (1135)96.6\% & (164)94.2\% & 0.180 & $1459(94.4 \%)$ & $1261(95 \%)$ & $198(92 \%)$ & 0.057 \\
\hline Poor (Family PIR<1.0) & $167(11.9 \%)$ & (144)10.9\% & (23)12.6\% & 0.706 & $256(9.4 \%)$ & $226(9.9 \%)$ & $30(7.4 \%)$ & 0.320 \\
\hline Total energy $(\mathrm{kcal} / \text { day })^{\mathrm{a}}$ & $1736.52(25.43)$ & $1727.63(25.06)$ & $1780.84(68.69)$ & 0.443 & $1929.58(31.28)$ & $1905.28(36.1)$ & 2047.49(81.9) & 0.155 \\
\hline Total protein $(\mathrm{g} / \text { day })^{\mathrm{a}, \mathrm{b}}$ & $76.33(0.73)$ & $76.18(0.88)$ & $77.08(1.87)$ & 0.684 & $78.48(0.74)$ & $78.5(0.92)$ & $78.41(0.57)$ & 0.983 \\
\hline Relative protein $(\mathrm{g} / \mathrm{kg} / \text { day })^{\mathrm{a}, \mathrm{b}}$ & $1.03(0.02)$ & $1.03(0.02)$ & $1.02(0.03)$ & 0.808 & $1.02(0.01)$ & $1.03(0.01)$ & $0.96(0.04)$ & 0.133 \\
\hline
\end{tabular}

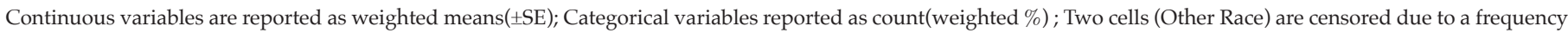

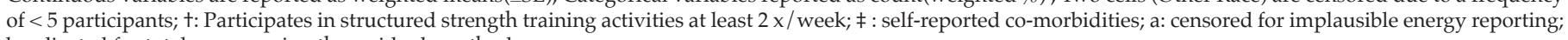
b. adjusted for total energy using the residuals method

\section{Statistical Analysis}

Consistent with NHANES analytic guidelines, we combined the two survey cycles containing grip strength measures (2011-2012 and 2013-2014) into a single dataset, which represented the Grip Strength Cohort. We applied variance estimation weights to account for the complex survey design of NHANES. Obesity was considered a comorbidity and derived using the body mass index threshold of $\geq 30 \mathrm{~kg} / \mathrm{m} 2$. In our analytic samples only the prevalence of chronic obstructive pulmonary diseases (COPD) exhibited urban/rural differences, therefore, we chose to define comorbidity burden as composite, ordinal variable, ranging from zero to $\geq 3$ comorbidities. To account for implausible energy reporting, we censored participants who reported less than 500 or more than 3500 kcals/day, resulting in approximately $6 \%$ of participants being censored from nutrient analyses (23).

To characterize the prevalence of slow gait speed, grip strength-defined sarcopenia and participant characteristics, we report count (weighted \%) for the overall and urban-rural stratified dichotomous measures. To assess urban vs. rural differences in baseline characteristics, we simulated weighted t-tests using a bivariate regression model for continuous variables and performed Pearson chi-square for categorical variables. A threshold of $p<0.05$ was considered significant. Stata v.14 (College Station, TX) was used for all statistical analyses.

To evaluate the associations between rural residency status, total energy, total protein as well as demographic, physical activity and comorbidity variables on continuous measures of gait speed and grip strength, we created sequentially-adjusted linear regression models. Model 1 assessed the unadjusted association between rural residency status and the outcomes. Model 2 was adjusted for total energy and energy-adjusted protein, and Model 3 was further adjusted for age, sex, race/Hispanic origin, number of comorbidities and physical activity. 


\section{Results}

Participant baseline characteristics for each cohort are presented in Table 1. The proportion of participants residing in rural areas was $16.7 \%$ and $19.4 \%$ in the Gait Speed and Grip Strength cohorts, respectively. Urban and rural participants were similar in age, sex, body mass index and number of comorbidities. Race/Hispanic origin and participation in strength training (in the Gait Speed cohort) were the only baseline characteristics exhibiting statistically-significant urban-rural differences. Total energy, total protein and protein adjusted for body mass (relative protein) intakes were similar between urban and rural participants, in both cohorts.

\section{Muscle weakness and slow gait speed prevalence}

Rural NHANES participants exhibited higher absolute grip strength and a lower prevalence of GripBMI weakness compared to urban participants (Table 2). Rural participants demonstrated an approximately $2 \mathrm{~kg}$. higher absolute grip strength and an $8.2 \%$ lower prevalence of GripBMI weakness compared to urban participants. No urban-rural differences in absolute gait speed or the prevalence slow gait speed were observed.

\section{Factors associated with gait speed}

Table 3 reports the results of the regression analysis for predictors of absolute gait speed. Rural residency status was not associated with gait speed in any models. Total energy was associated with slower gait speed in Model 2 , but adjustment for demographic, physical activity and comorbidity covariates in Model 3, rendered the association insignificant. All of the covariates in Model 3 exhibited significant, positive associations with gait speed, except for self-reported participation in strength training activities ( $\geq 2 x /$ week), which was associated with slower gait speed. Surprisingly, comorbidity burden was associated with faster gait speed, and a dose-response relationship was observed.

\section{Factors associated with grip strength}

Table 4 reports the results of the regression analysis for predictors of absolute grip strength. In the unadjusted model, rural residency status was associated with absolute grip strength but only explained $0.6 \%$ of the variability. Adjusting for total energy and energyadjusted protein attenuated this association and explained about $15 \%$ of the variability in grip strength. Fully adjusting for covariates revealed that total energy was associated with higher absolute grip strength. Age and being female were associated with lower grip strength. In this cohort, comorbidity burden was not associated with grip strength in these data.

\section{Discussion}

We sought to examine urban-rural differences in the prevalence of grip strength-defined muscle weakness and slow gait speed in a sample of older adults. We found that rural participants exhibited higher absolute grip strength and lower prevalence of muscle weakness compared to their urban counterparts. This is the first study exploring such differences using the NHANES and is of clinical importance, as the influence of urbanization status on muscle weakness, a primary indicator of sarcopenia in older adults is not well understood.

Table 2

Prevalence of Slow Gait Speed and Grip Weakness for each NHANES cohort

\begin{tabular}{|c|c|c|c|c|}
\hline & \multicolumn{4}{|c|}{ NHANES 2001-2002 } \\
\hline & Overall & Urban & Rural & p-value \\
\hline & $\mathrm{n}=1,350$ & $\mathrm{n}=1176(83.3 \%)$ & $\mathrm{n}=174(16.7 \%)$ & \\
\hline Gait speed (m/s) & $1.17(0.02)$ & $1.16(0.02)$ & $1.18(0.04)$ & 0.732 \\
\hline Slow gait speed & $118(11.7 \%)$ & $100(11.1 \%)$ & $18(14.3 \%)$ & 0.229 \\
\hline Slow gait speed $(<0.8 \mathrm{~m} / \mathrm{s})$ & & & & \\
\hline
\end{tabular}

\begin{tabular}{|c|c|c|c|c|}
\hline & \multicolumn{4}{|c|}{ NHANES 2011-2014 } \\
\hline & Overall & Urban & Rural & p-value \\
\hline & $\mathrm{n}=1,573$ & $\mathrm{n}=1,362(80.7 \%)$ & $\mathrm{n}=211(19.4 \%)$ & \\
\hline Handgrip strength (kg.) & $32.13(0.43)$ & $31.75(0.45)$ & $33.73(0.48)$ & 0.001 \\
\hline $\operatorname{Grip}_{\text {вмI }}$ & $1.14(0.01)$ & $1.13(0.01)$ & $1.19(0.03)$ & 0.131 \\
\hline Grip $_{\text {(weak) }}$ & $418(21.2 \%)$ & $367(22.1 \%)$ & $51(17.4 \%)$ & 0.085 \\
\hline $\operatorname{GripBMI}_{\text {(weak) }}$ & $441(25.8) \%$ & $387(27.4 \%)$ & $54(19.2 \%)$ & 0.001 \\
\hline
\end{tabular}


Table 3

Associations for rural status and gait speed: NHANES 2001-2002; Gait Speed Cohort

\begin{tabular}{|c|c|c|c|c|c|c|c|c|c|}
\hline & \multicolumn{3}{|c|}{ Model I } & \multicolumn{3}{|c|}{ Model 2} & \multicolumn{3}{|c|}{ Model 3} \\
\hline & $\mathrm{B}( \pm \mathrm{SE})$ & {$[95 \% \mathrm{CI}]$} & p-value & $\mathrm{B}( \pm \mathrm{SE})$ & {$[95 \% \mathrm{CI}]$} & p-value & $\mathrm{B}( \pm \mathrm{SE})$ & {$[95 \% \mathrm{CI}]$} & p-value \\
\hline Rural & $0.01(0.04)$ & {$[-0.07,0.10]$} & 0.732 & $0.02(0.04)$ & {$[-0.07,0.12]$} & 0.607 & $0.00(0.04)$ & {$[-0.08,0.08]$} & 0.941 \\
\hline Total protein ${ }^{\mathrm{a}, \mathrm{b}}$ & -- & & & $0.000(0.000)$ & {$[-0.000,0,001]$} & 0.203 & $0.001(0.001)$ & {$[-0.000,0.002]$} & 0.251 \\
\hline Total energy ${ }^{a}$ & -- & & & $0.000(0.000)^{* * *}$ & {$[-0.0002,-0,0002]$} & $<0.001$ & $0.0000(0.0000)$ & {$[-0.0001,0.0000]$} & 0.056 \\
\hline Age & -- & & & -- & & & $0.02(0.003)^{* * *}$ & {$[0.02,0.03]$} & $<0.001$ \\
\hline Female & -- & & & -- & & & $0.06(0.02)^{* *}$ & {$[0.02,0.10]$} & 0.008 \\
\hline \multicolumn{10}{|l|}{ Race/Ethnicity } \\
\hline $\begin{array}{l}\text { Mexican/Other His- } \\
\text { panic }\end{array}$ & -- & & & -- & & & $0.19(0.06)^{* *}$ & {$[0.07,0.30]$} & 0.004 \\
\hline Non-Hispanic White & -- & & & & --referent-- & & & --referent-- & \\
\hline Non-Hispanic Black & -- & & & -- & & & $0.17(0.06)^{* *}$ & {$[0.05,0.29]$} & 0.009 \\
\hline Other & -- & & & -- & & & $0.14(0.05)^{* *}$ & {$[0.04,0.24]$} & 0.008 \\
\hline Strength training & & & & -- & & & $-0.08(0.04)^{*}$ & {$[-0.16,-0.01]$} & 0.034 \\
\hline \multicolumn{10}{|l|}{ \# of co-morbidities $¥$} \\
\hline 0 & -- & & & -- & & & & & \\
\hline 1 & -- & & & -- & & & $0.08(0.02)^{* *}$ & {$[0.03,0.12]$} & 0.001 \\
\hline 2 & -- & & & -- & & & $0.19(0.05)^{* *}$ & {$[0.08,0.30]$} & 0.002 \\
\hline$\geq 3$ & -- & & & -- & & & $0.29(0.05)^{* * *}$ & {$[0.19,0.40]$} & $<0.001$ \\
\hline $\mathrm{R}^{2}$ & & 0.0001 & & & 0.03 & & & 0.22 & \\
\hline
\end{tabular}

Table 4

Associations for rural status and absolute grip strength: NHANES 2011-2014; Grip Strength Cohort

\begin{tabular}{|c|c|c|c|c|c|c|c|c|c|}
\hline & \multicolumn{3}{|c|}{ Model I } & \multicolumn{3}{|c|}{ Model 2} & \multicolumn{3}{|c|}{ Model 3} \\
\hline & $\mathrm{B}( \pm \mathrm{SE})$ & {$[95 \% \mathrm{CI}]$} & p-value & $\mathrm{B}( \pm \mathrm{SE})$ & {$[95 \% \mathrm{CI}]$} & $\mathrm{p}$-value & $\mathrm{B}( \pm \mathrm{SE})$ & {$[95 \% \mathrm{CI}]$} & $\mathrm{p}$-value \\
\hline Rural & $1.99(0.50)^{* *}$ & {$[0.92,3.05]$} & 0.001 & $2.18(1.29)$ & {$[-0.56,4.94]$} & 0.112 & $1.45(0.82)$ & {$[-0.29,3.19]$} & 0.097 \\
\hline Total protein ${ }^{\mathrm{a}, \mathrm{b}}$ & -- & & & $0.10(0.03)^{\star \star}$ & {$[0.04,0.16]$} & 0.004 & $0.01(0.01)$ & {$[-0.02,0.04]$} & 0.472 \\
\hline Total energy ${ }^{\mathrm{a}}$ & -- & & & $0.006(0.001)^{\star * *}$ & {$[-0.000,-0000]$} & $<0.001$ & $0.001(0.000)^{* *}$ & {$[0.001,0.002]$} & 0.003 \\
\hline Age & -- & & & -- & & & $-0.46(0.03)^{* * *}$ & {$[-0.52,-0.39]$} & $<0.001$ \\
\hline Female & -- & & & -- & & & $-14.83(0.51)^{\star \star * *}$ & {$[-15.92,-13.73]$} & $<0.001$ \\
\hline \multicolumn{10}{|l|}{ Race/Ethnicity } \\
\hline Mexican/Other Hispanic & -- & & & -- & & & $-3.35(0.50)^{* * *}$ & {$[-4.41,-2.91]$} & $<0.001$ \\
\hline Non-Hispanic White & -- & & & & & & & --referent-- & \\
\hline Non-Hispanic Black & -- & & & -- & & & $2.09(1.05)$ & {$[-0.14,4.32]$} & 0.064 \\
\hline Other & - & & & -- & & & $-2.51(0.81)^{\star \star}$ & {$[-4.24,-0.79]$} & 0.007 \\
\hline Sedentary time $(\mathrm{min} / \mathrm{d}) \dagger$ & & & & - & & & $-0.002(0.001)$ & {$[-0.01,0.00]$} & 0.061 \\
\hline \multicolumn{10}{|l|}{ \# of co-morbidities $¥$} \\
\hline 0 & -- & & & -- & & & & & \\
\hline 1 & -- & & & -- & & & $-0.05(0.80)$ & {$[-1.75,1.65]$} & 0.954 \\
\hline 2 & -- & & & -- & & & $0.21(0.57)$ & {$[-1.01,1.43]$} & 0.715 \\
\hline$\geq 3$ & -- & & & -- & & & $-1.64(1.00)$ & {$[-3.77,0.48]$} & 0.120 \\
\hline $\mathrm{R}^{2}$ & & 0.006 & & & 0.15 & & & 0.68 & \\
\hline
\end{tabular}

Model I: unadjusted for covariates; Model 2: Model I, adjusted for total energy and protein; Model 3: Model 2, adjusted for age, sex and race/Hisp. Origin \# of comorbidities and strength training; a. censored to remove implausible energy reports; b. adjusted for total energy, using the residuals method; ${ }^{*} \mathrm{p}<0.05 ;{ }^{* *} \mathrm{p}<0.01 ;{ }^{* * *} \mathrm{p}<0.001$ 
Contrary to our initial hypothesis that, because of rural health inequities, rural older adults would exhibit a higher prevalence of muscle weakness and slow gait speed (i.e., would be weaker and exhibit lower function). However, we did not observe health inequities between urban and rural participants. Furthermore, urban-rural differences in several important baseline characteristics, such as age, sex, nutritional factors and socioeconomic were absent in both cohorts. This may have been a result of sampling issues within the urban-rural indicator variable, which is part of the restricted data program in the NHANES. It may be such that the rural impoverished older adults without health insurance may have been underrepresented, or their richer, more insured counterparts, overrepresented in this restricted NHANES sample. Historically, most reports on urban-rural health disparities have been from studies observing adults across wide age-ranges, not limited to older adults(24). For example, Fan, et. al. (25) reports urban-rural disparities in self-reported physical activity in 20-75 yr. old adults participating in the NHANES. Moreover, such studies have reported that these differences vary across regions of the United States. Importantly, our analysis did not compare urban-rural differences by geographic differences, rather by demographic differences.

Our findings agree with studies assessing the grip strength of older men and women from India (26) and Japanese women (27). In contrast, we are aware of one study reporting a higher prevalence of sarcopenia in rural compared to urban older Chinese adults ( $\geq 60$ yrs.) (28). A common thread in these previous studies is the heterogeneity among the study methods, including definitions of sarcopenia, measures used, race and behaviors of study populations, which may render comparisons spurious.

Estimates from the fully-adjusted grip strength model suggest that, for every $100 \mathrm{kcal}$ increase in energy intake, we would expect to see absolute grip strength increase by $0.1 \mathrm{~kg}$. This parallels findings from Okamura (29) who reported that, in older adults with diabetes, every $100 \mathrm{kcal}$ increase in total energy, was accompanied by $11 \%$ lower odds of having sarcopenia. Similarly, in a populationbased study using NHANES data, Smit (30) reported that higher energy intakes, but not protein were associated with lower odds of having frailty, defined using the Fried frailty phenotype, of which, grip strength and gait speed are components.

An unexpected finding was that comorbidity burden was similar among urban and rural participants. This may, in part, explain why we did not observe betweengroup differences in gait speed. A study by Ortiz (31) employed a similar comorbidity classification to the present study and, in unadjusted models, they reported slower gait speeds across increasing numbers of comorbidities. Importantly, these associations were attenuated when adjusting for demographic covariates, rendering only the $\geq 3$ comorbidities category associated with gait speed. A fairly large body of evidence suggests that urban-rural differences in common comorbidities, such as heart disease (7), obesity (24), and smoking status (32). While it is unclear exactly why data in the present study did not exhibit urban-rural differences in comorbidity burden, it may involve methodological differences in the definition of comorbidity burden. Four of the five chronic health conditions used to synthesize the comorbidity variable in the present study were derived from self-reported data.

\section{Strengths and Limitations}

This study has several strengths. This is the first study comparing the prevalence and characteristics of grip weakness and slow gait speed between urban and rural older adults using the NHANES. We used recently-published grip strength and gait speed cut points published by the SDOC to define outcomes. These cut points have been validated as predictors of adverse health outcomes associated with sarcopenia, which, we believe enhances the clinical relevance of this report. The present study also has some limitations. The NHANES dataset used for the gait speed analysis is twenty years old, and the sample consisted of non-institutionalized older adults who were relatively high-functioning, thus, our findings may not reflect currently-institutionalized older adults. The cross-sectional nature of this study prevents any inferences of causation between exposures and outcomes. Another limitation involves the comorbidity variable used in these analyses. Four of the five source variables defining comorbidity burden used self-reported medical conditions. Compared to using physician-diagnosed medical conditions, this approach may be prone to misclassification bias.

\section{Conclusions}

These findings provide novel insight regarding the influence of rural residency status on indicators of sarcopenia. In this sample of older adults, rural participants were stronger, but exhibited similar gait speed compared to their urban counterparts. No urban-rural differences in nutritional or socioeconomic factors were observed in this sample. Consistent with previous reports, total energy was positively-associated with higher grip. Unraveling the interaction between urbanization level and nutritional factors, strength and function will help clinicians, researchers and public health policymakers identify efficient therapeutic targets to treat sarcopenia and associated health outcomes.

Conflict of interest: We report no conflicts of interest in this research project. 
Ethical standard: This study was approved by Tufts University SocialBehavioral Educational Research Review Board; Study \# 1811045.

Acknowledgments: Special thanks to James Davis, Ph.D. Corey O'hara. Ph.D., Yi Zhao, MS, Aziz authored and conceptualized the study design, conducted the data analysis and manuscript preparation. Reid conceptualized the study design, critically reviewed the manuscript and approved the final version. Batsis conceptualized the study design, reviewed, and edited analytic frameworks, and contributed to the final manuscript preparation. Fielding conceptualized the study design, critically reviewed the manuscript and approved the final version.

Funding sources: This project was supported by the USDA under agreement No. 58-8050-9-004. The opinions stated in this paper do not represent the opinions of the USDA. JAB is funded in part by the National Institute on Aging of the National Institutes of Health under Award Number K23AG051681. The content is solely the responsibility of the authors and does not necessarily represent the official views of the National Institutes of Health or other sponsor.

\section{References}

1. R. A. Fielding et al., Sarcopenia: an undiagnosed condition in older adults Current consensus definition: prevalence, etiology, and consequences. International working group on sarcopenia. J Am Med Dir Assoc 2011;12, 249-256.

2. A. J. Cruz-Jentoft et al., Prevalence of and interventions for sarcopenia in ageing adults: a systematic review. Report of the International Sarcopenia Initiative (EWGSOP and IWGS). Age Ageing 2014;43, 748-759.

3. J. A. Batsis et al., Variation in the prevalence of sarcopenia and sarcopenic obesity in older adults associated with different research definitions: dualenergy X-ray absorptiometry data from the National Health and Nutrition Examination Survey 1999-2004. J Am Geriatr Soc 2013;61, 974-980.

4. P. M. Cawthon et al., Establishing the Link Between Lean Mass and Grip Strength Cut-points With Mobility Disability and Other Health Outcomes: Proceedings of the Sarcopenia Definition and Outcomes Consortium Conference. J Gerontol A Biol Sci Med Sci, (2019).

5. 2010 Census Urban and Rural Classification and Urban Area Criteria (2010 https: / / www.census.gov/programs-surveys/geography / guidance/geoareas / urban-rural/2010-urban-rural.html).

6. E. Moy et al., Leading Causes of Death in Nonmetropolitan and Metropolitan Areas- United States, 1999-2014. MMWR Surveill Summ 2017;66, 1-8.

7. A. Kulshreshtha, Goyal, A, Urban-Rural Differences in Coronary Heart Disease Mortality in the United States: 1999-2009. Public Health Reports 2014;129.

8. S. Studenski et al., Gait speed and survival in older adults. JAMA 2011;305, 50-58.

9. S. Bhasin et al., Sarcopenia Definition: The Position Statements of the Sarcopenia Definition and Outcomes Consortium. J Am Geriatr Soc, (2020).

10. D. D. Ingram, S. J. Franco, 2013 NCHS Urban-Rural Classification Scheme for Counties. Vital Health Stat 2014;2, 1-73

11. NHANES Muscle Strength Procedures Manual (2013 https:/ / wwwn.cdc gov/nchs/data/nhanes/2013-2014/manuals/muscle_strength_2013.pdf).

12. A. F. Subar et al., The Automated Self-Administered 24-hour dietary recall (ASA24): a resource for researchers, clinicians, and educators from the National Cancer Institute. J Acad Nutr Diet 2012;112, 1134-1137.

13. A. J. Moshfegh et al., The US Department of Agriculture Automated MultiplePass Method reduces bias in the collection of energy intakes. Am J Clin Nutr 2008;88, 324-332.
14. J. Shlisky et al., Nutritional Considerations for Healthy Aging and Reduction in Age-Related Chronic Disease. Adv Nutr 2017;8, 17-26.

15. D. K. Houston et al., Dietary protein intake is associated with lean mass change in older, community-dwelling adults: the Health, Aging, and Body Composition (Health ABC) Study. Am J Clin Nutr 2008;87, 150-155.

16. H. Campos et al., Nutrient intake comparisons between Framingham and rural and Urban Puriscal, Costa Rica. Associations with lipoproteins, apolipoproteins, and low density lipoprotein particle size. Arterioscler Thromb 1991;11, 1089-1099

17. W. C. Willett, G. R. Howe, L. H. Kushi, Adjustment for total energy intake in epidemiologic studies. Am J Clin Nutr 1997;65, 1220S-1228S; discussion 1229S-1231S.

18. A. J. Cruz-Jentoft et al., Sarcopenia: European consensus on definition and diagnosis: Report of the European Working Group on Sarcopenia in Older People. Age Ageing 2010;39, 412-423.

19. J. Mesinovic, A. Zengin, B. De Courten, P. R. Ebeling, D. Scott, Sarcopenia and type 2 diabetes mellitus: a bidirectional relationship. Diabetes Metab Synd Obes 2019;12, 1057-1072 .

20. G. Bahat et al., Relation between hand grip strength, respiratory muscle strength and spirometric measures in male nursing home residents. Aging Male 2014;17, 136-140.

21. R. Roubenoff, R. A. Roubenoff, L. M. Ward, S. M. Holland, D. B. Hellmann Rheumatoid cachexia: depletion of lean body mass in rheumatoid arthritis. Possible association with tumor necrosis factor. J Rheumatol 1992;19, 15051510 .

22. M. Bonnefoy, T. Gilbert, [Body composition and comorbidity in the elderly]. Geriatr Psychol Neuropsychiatr Vieil 2015;13 Suppl 1, 29-36.

23. J. J. Rhee et al., Comparison of methods to account for implausible reporting of energy intake in epidemiologic studies. Am J Epidemiol 2015;181, 225-233.

24. P. D. Patterson, C. G. Moore, J. C. Probst, J. A. Shinogle, Obesity and physical inactivity in rural America. J Rural Health 2004;20, 151-159.

25. J. X. Fan, M. Wen, L. Kowaleski-Jones, Rural-urban differences in objective and subjective measures of physical activity: findings from the National Health and Nutrition Examination Survey (NHANES) 2003-2006. Prev Chronic Dis 2014;11, E141.

26. P. Dhara, P. Sengupta, S. De, Hand Grip Strength of Older Persons in Relation to Body Dimensions and Nutritional Status. Journal of Indian Academy of Geriatrics (2011).

27. R. A. Carvalho Sampaio, P. Y. Sewo Sampaio, M. Yamada, M. Ogita, H. Arai, Urban-rural differences in physical performance and health status among older Japanese community-dwelling women. Journal of Clinical Gerontology and Geriatrics 2012;3, 127-131.

28. L. Gao et al., Prevalence of Sarcopenia and Associated Factors in Chinese Community-Dwelling Elderly: Comparison Between Rural and Urban Areas. J Am Med Dir Assoc 2015;16, 1003.e1001-1006.

29. T. Okamura et al., Shortage of energy intake rather than protein intake is associated with sarcopenia in elderly patients with type 2 diabetes: A crosssectional study of the KAMOGAWA-DM cohort. J Diabetes 2019;11, 477-483.

30. E. Smit, K. M. Winters-Stone, P. D. Loprinzi, A. M. Tang, C. J. Crespo, Lower nutritional status and higher food insufficiency in frail older US adults. Br J Nutr 2013;110, 172-178.

31. P. J. Ortiz et al., Effect of multimorbidity on gait speed in well-functioning older people: A population-based study in Peru. Geriatr Gerontol Int 2018;18, 293-300.

32. K. E. Weaver, N. Palmer, L. Lu, L. D. Case, A. M. Geiger, Rural-urban differences in health behaviors and implications for health status among US cancer survivors. Cancer Causes Control 2013;24, 1481-1490. 